\title{
Traduire
}

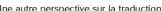

Revue française de la traduction

$218 \mid 2008$

De traduction en retraduction

\section{Traduire et retraduire Sense and Sensibility ou comment faire aimer Austen en français}

\section{Valérie Cossy}

\section{(2) OpenEdition}

1 Journals

Édition électronique

URL : http://journals.openedition.org/traduire/897

DOI : $10.4000 /$ traduire.897

ISSN : 2272-9992

Éditeur

Société française des traducteurs

Édition imprimée

Date de publication : 15 juin 2008

Pagination : 43-64

ISSN : 0395-773X

\section{Référence électronique}

Valérie Cossy, «Traduire et retraduire Sense and Sensibility ou comment faire aimer Austen en

français », Traduire [En ligne], 218| 2008, mis en ligne le 15 juin 2008, consulté le 10 décembre 2020.

URL : http://journals.openedition.org/traduire/897 ; DOI : https://doi.org/10.4000/traduire.897 


\section{Traduire et retraduire Sense and Sensibility ou comment faire aimer Austen en français}

\section{VALÉRIE COSSY}

Cet article se propose d'étudier le sort de Jane Austen en français à travers les traductions et retraductions de Sense and Sensibility afin, peut-être, de dégager quelques éléments de réponse à la question frustrante que se posent les admirateurs francophones de la romancière (qui l'ont lue en anglais) : pourquoi est-elle si mal comprise, voire méprisée, en français et, accessoirement, pourquoi est-il impossible de faire partager son enthousiasme à ses amis en leur proposant une version française de ses romans qui lui rende justice? Tout en sachant que la critique est aisée et l'art beaucoup plus difficile, nous aimerions proposer des pistes de réflexions aux futurs retraducteurs d'Austen.

Sense and Sensibility fut le premier roman qu'Austen réussit à faire publier, en 1811, et le troisième à être traduit en français après Pride and Prejudice et Mansfield Park, qui, en 1813 et 1815 respectivement, parurent en version abrégée et en feuilleton dans la Bibliothèque britannique, un périodique rédigé et publié à Genève et largement diffusé en France (Cossy ; Bickerton). La première traduction de Sense and Sensibility parut également en 1815 et fut publiée à Paris, chez Arthus Bertrand. Ce dernier était l'éditeur attitré d'Isabelle de Montolieu, romancière à succès, qui, tout en résidant à Lausanne, publiait à Paris où elle touchait un large public dans les cabinets de lecture grâce à ses romans, pour la plupart adaptés de l'allemand ou de l'anglais (Trunel : 63-113 ; Parent-Lardeur : 172). Montolieu traduisit, ou plutôt, adapta dans la veine qui avait fait son succès non pas un mais deux romans d'Austen : Sense and Sensibility dont il sera question ici, sous le titre Raison et sensibilité ou les deux manières d'aimer, ainsi 
que Persuasion, roman qui parut à la fin de 1817 en Angleterre peu après la mort de l'auteur, et qu'elle traduisit en 1821 sous le titre La Famille Elliot ou l'ancienne inclination. Tous les romans d'Austen furent traduits en peu de temps en français, en deux vagues successives, entre 1813 et 1824 . Ces deux vagues suivent de près les adaptations de Montolieu, et il est raisonnable de penser que la traductrice elle-même romancière à succès - plus que l'auteur original encore inconnu, attira l'attention des éditeurs et du public français sur l'œuvre d'Austen. À la suite de Raison et sensibilité paraissent en 1816 une traduction intégrale de Mansfield Park chez Dentu (Le parc de Mansfield ou les trois cousines, traduit par Henri Vilmain) et une traduction anonyme d'Emma chez Arthus Bertrand (La Nouvelle Emma ou les caractères anglais du siècle). Après La Famille Elliot, deux nouvelles traductions de Pride and Prejudice voient le jour en 1822 : une anonyme publiée chez Paschoud et une par Eloïse Perks chez Maradan. Enfin, dernier roman à être traduit, Northanger Abbey paraît en 1824 chez Pigoreau sous le titre L'Abbaye de Northanger dans une traduction de Hyacinthe de Ferrière (Gilson; Bour). Même si les autres traducteurs du début du XIXe siècle sont un peu moins infidèles que Montolieu, tous néanmoins inscrivent leur travail dans son sillage. Dans le paratexte des premières traductions, en effet, Jane Austen n'est pas "l'auteur de Pride and Prejudice ", formule qui la désigne en Angleterre, mais "l'auteur que Mme de Montolieu a fait connaître ». Dans le jargon des catalogues, cela signifie tout simplement qu'Austen est rangée au rayon du roman sentimental. En cela l'impact de Montolieu sur sa réception en France au XIXe siècle a été déterminant en occultant la dimension réaliste et ironique de l'œuvre.

Pour tenter de comprendre le sort de Jane Austen en France, nous avons retenu Sense and Sensibility car, d'Isabelle de Montolieu à la Pléiade, ce roman offre un panorama intéressant de traduction et de retraductions. Ayant fait l'objet d'une première traduction - "traduction libre" pour être précis - par la traductrice de renom qui fonde la réputation d'Austen en France au XIXe siècle, il est retraduit en 2000 par l'angliciste universitaire Pierre Goubert sous le 
titre Le Coeur et la Raison, dans le cadre le plus sérieux que l'on puisse imaginer, celui de la Bibliothèque de la Pléiade qui, en France, fonctionne

comme

instance de consécration des ouvres et des auteurs, auxquels elle confere le statut de classiques. En traduction française, Sense and Sensibility se retrouve donc aux deux extrémités d'un spectre chronologique et typologique, qui va du vivant de l'auteur à l'époque contemporaine et de la littérature comme divertissement à la "grande" Littérature. Nous nous arrêterons également sur deux autres retraductions, celle par Jules Castier parue chez Marguerat à Lausanne en 1948 (Le Cour et la Raison) et celle de Jean Privat, Raison et sentiments, qui remonte également à 1948 mais qui joue, aujourd'hui encore, un rôle capital dans la diffusion de Sense and Sensibility en français puisqu'il s'agit du texte de l'édition de poche 10/18. Un texte manque pour que notre panorama des retraductions soit complet, celui d'Eugène Rocart (Raison et Sensibilité) paru à Bruxelles aux Éditions de la Boétie en 1945 et que nous n'avons pas pu nous procurer. Quoi qu'il en soit, le but ici n'est pas de nous livrer à une comparaison exhaustive des textes traduits mais, à partir de quelques exemples, de cerner ce qui, depuis le XIXe siècle, fait obstacle au transfert d'Austen en français. Sans minimiser la difficulté de l'acte traducteur lui-même, nous proposons comme hypothèse que le nœud du problème réside en premier lieu dans le contexte qui préside à l'interprétation des textes. C'est à l'élucidation de cette notion de contexte et de son contenu que nous aimerions consacrer cet article pour saisir les faiblesses mais aussi les enjeux des traductions et retraductions d'Austen en français.

Que peut-on dire des retraductions de Sense and Sensibility ? Si l'on s'en remet à la critique des traductions et retraductions telle qu'Antoine Berman la conçoit, celles-ci s'inscrivent dans un processus général de " translation " des œuvres, de leur passage de ce qu'il appelle une "langue culture " à une autre. Une traduction ou une retraduction constituent chacune un "moment» de ce processus qu'est la translation, qui, idéalement, devrait aboutir au dévoilement de l'œuvre originale dans la « langue culture » réceptrice : 
On voit aisément que le sens de cette translation est la "révélation" d'une ouvre étrangère dans son être propre à la culture réceptrice. La "révélation" pleine et entière de cette cuvre est elle-même l'œuvre de la traduction, et d'elle seule. Et elle n'est possible que si la traduction est "vraie ». (Berman : 57).

Notre approche s'inscrit dans la perspective développée par Berman dans la mesure où nous constatons que la "révélation " d'Austen en français n'a pas encore eu lieu. En cela, notre commentaire des traductions et retraductions ne sera pas neutre, mais sous-entend qu'une meilleure traduction reste à faire car la «translation " d'Austen dans la "langue culture» française n'a pas encore abouti, preuve en est la " régression " que constitue la publication en 1996 par l'Archipel et en 2006 en "archipoche" de la traduction de 1815 d'Isabelle de Montolieu sous le titre Raison et Sentiments. Le concept de " translation " de Berman a aussi l'avantage d'élargir la critique des traductions et retraductions à la réception critique : le processus est en effet " accompagné, soutenu par tout un travail critique ", dit-il, qui contribue avec les retraductions de l'œuvre à son dévoilement (Berman : 57). Or la situation d'Austen en français nous paraît relever d'un profond décalage entre les réceptions de l'œuvre et les traditions critiques de part et d'autre de la Manche. Et, à moins d'une ouverture de la part des traducteurs à ce qui se dit d'Austen en "langue culture " anglaise, de Londres à New York et de New York à Melbourne, il nous paraît peu probable de voir s'améliorer la compréhension et la restitution d'Austen en français, " langue culture " dans laquelle elle reste prisonnière d'un nombre impressionnant de malentendus culturels et/ou sexistes.

La livraison de juin 2008 du Magazine littéraire consacré aux " romancières anglaises" (en pink dans le titre) en offre encore un exemple désolant. Austen figure en couverture sur une théière aux côtés de Virginia Woolf - affublée pour la circonstance d'un fard à paupières indigne de son élégance légendaire - et de Zadie Smith. Que ce soit en couverture ou à l'intérieur, Austen y apparaît inséparable 
d'une théière sous les traits de la gravure de Lizars - colorisée pour la circonstance -, elle-même effectuée d'après le portrait d'Andrews et commanditée par James-Edward Austen-Leigh pour servir de frontispice à son Memoir of Jane Austen (1870). Réalisé à partir d'une esquisse de Cassandra Austen, ce portrait posthume était jugé peu ressemblant par la famille, mais il contribua pleinement à l'élaboration d'Austen en mythe victorien (Sutherland : xiv-xivi). À lire ensuite les propos que lui consacre Christine Jordis, on a vraiment l'impression que, au sein de la culture éditoriale française qui produit des "romancières anglaises " sur le mode collectif, Austen demeure effectivement à l'état de mythe victorien. Ses romans, placés à côté de ceux de Barbara Pym et Anita Brookner, sont qualifiés de "parfaitement traditionnels", relevant $d^{\prime}$ '" une tradition des écrivains de l'enfermement" qui "se situent plus loin du roman féministe que certaines œuvres écrites par des hommes ". Pour Jordis, dans les romans d'Austen « la leçon est d'avance entendue ": " $S$ 'adapter et non défier, se conformer, non s'imposer, faire preuve de sagesse plutôt que d'affirmer son désir ». En outre, toujours selon Jordis, le monde et ses lois seraient, pour les héroïnes d'Austen, "immuables» (Jordis : 56-57). Les admirateurs d'Anne Elliot et les commentateurs du scepticisme d'Austen apprécieront!

Si enfermement il y a, c'est celui, pervers, dans lequel la «langue culture " française, à l'instar du Magazine littéraire, a jusqu'ici confiné Austen pour des raisons que j'avoue difficiles à cerner. On s'attendrait notamment à ce que Christine Jordis, "directrice du domaine anglosaxon chez Gallimard ", produise un commentaire qui donne envie de lire Austen et, éventuellement, de se procurer le volume de la Pléiade. Or ses propos sont carrément dissuasifs. Pour le moins, cette vision négative de l'œuvre peine à expliquer son audience planétaire : la résignation comme clé du succès ? On en doute. En qualifiant sommairement ses romans de "traditionnels ", cette doxa française réductrice opère également une confusion entre la forme et le fond. On ignore ou feint d'ignorer les avancées stylistiques que propose son art du roman, notamment son usage novateur, fluide et généralisé, du style indirect 
libre qui fait de son œuvre une étape importante dans le développement du genre romanesque en Angleterre. Quant au fond, les lecteurs de l'œuvre en anglais savent bien que les personnages favoris d'Austen ne sont justement pas ceux qui se plient soit à la tradition soit aux réalités extérieures. Austen livre même à notre sagacité une galerie impressionnante de faiseurs de courbettes, d'hypocrites matérialistes, de fétichistes de la dignité aristocratique, personnages dénués de sentiment et d'imagination. Le général Tilney, John et Fanny Dashwood, Mr Collins, Mrs Bennet, Caroline Bingley, l'abominable Mrs Norris, Rushworth, les sœurs Bertram, Mr et Mrs Elton, Sir Walter Elliot, tous incarnent une norme étroite et répressive que dénoncent les romans et contre laquelle s'élèvent les désirs, l'intelligence et l'individualisme "romantique " des héroïnes de même que leur exigence morale fondée sur une notion du "devoir" héritée des Lumières. L'article du Magazine littéraire recouvre aussi d'un épais brouillard l'apport de la critique féministe à la compréhension d'Austen en utilisant de manière très empirique - c'est un euphémisme - les termes "féminin " et "féministe ». Il ignore délibérément les ressources et les subtilités de cet apport critique qui, de Margaret Kirkham à Gary Kelly, en passant par Janet Todd et Claudia Johnson ont mis en lumière l'importance et la complexité des enjeux féministes dans les romans d'Austen et de ses contemporaines. Enfin, absence accablante qui est à l'image des traductions existantes, aucune mention n'est faite par Jordis de l'humour ou de l'ironie d'Austen, alors même que c'est par l'humour et par l'ironie qu'aujourd'hui encore elle s'impose prioritairement au lectorat anglophone.

Dans ses réflexions sur l'interprétation littéraire, par exemple, Stanley Fish s'appuie sur le statut d'Austen en tant que "master ironist" comme l'exemple le plus parlant qu'il puisse imaginer d'un de ces consensus critiques fondant les "communautés interprétatives". D'après Fish, le sens d'un texte ne lui est pas intrinsèque mais dépend d'une communauté de lecteurs partageant des valeurs communes de même qu'une vision implicite des œuvres et des auteurs. Un roman comme Pride and Prejudice, dit-il, peut théoriquement donner lieu à 
plusieurs interprétations radicalement opposées. Mais ces interprétations n'auront pas lieu car l'ironie d'Austen fait l'objet d'un consensus inébranlable qui préside implicitement à la lecture de ses romans :

Again the point is that while there are always mechanisms for ruling out readings, their source is not the text but the presently recognized narrative strategies for producing the text. It follows, then, that no reading, however outlandish it might appear, is inherently an impossible one.

La raison pour laquelle personne ne prendra Mr Collins au sérieux, par exemple, ne réside pas dans le fait que le texte signale de manière non équivoque son statut ironique, mais dans le fait que " it is unlikely that anyone who is now working in the Austen industry begins with an assumption other than the assumption that the novelist is a master ironist » (Fish : 447).

Les propos de Fish sur Austen nous intéressent à plus d'un titre. Tout d'abord, le fait même que Fish l'utilise comme exemple indique à quel point son ironie constitue, en effet, un consensus massif et incontournable pour tous ses lecteurs de langue anglaise. Ensuite, sa notion de "communauté interprétative" et sa perception de l'ironie d'Austen comme dépendant, précisément, d'une communauté interprétative peuvent nous aider à saisir les enjeux spécifiques de la "translation" d'Austen en français dans un sens légèrement décalé par rapport à celui où l'entend Berman. Si la "révélation " d'Austen au public francophone n'a pas encore eu lieu, c'est d'une part parce que l'ironie d'Austen rend instable le sens du texte mais également parce que la révélation d'Austen ne peut consister dans le "dévoilement" d'un sens caché, inhérent, non encore découvert, mais dans un rapprochement entre les communautés interprétatives. Les traductions et retraductions d'Austen de même que bon nombre de commentaires en français demeurent en effet à ce jour "outlandish": l'absence de propos sur son ironie doit frapper un lecteur britannique, américain ou australien comme un exotisme en décalage avec « sa " Jane Austen. L'objectif à atteindre par une retraduction d'Austen n'est pas 
l'élaboration d'un texte "juste", entreprise rendue d'autant plus impossible dès lors que l'on admet la présence de l'ironie, mais une traduction qui rapproche le texte français de la communauté interprétative anglophone. Que l'on nous entende bien : une traduction " outlandish " qui partirait du principe que Mr Collins est à prendre au sérieux ou que "l'enfermement " est en effet une valeur fondamentale de l'univers austenien ne serait pas fausse en elle-même. Elle serait simplement en décalage avec la perception de l'œuvre par la communauté interprétative anglophone. Or c'est le texte produit par cette communauté interprétative que les admirateurs d'Austen attendent encore de lire en français. Une retraduction qui serait au diapason de la communauté interprétative de " langue culture " anglaise clarifierait aux yeux du public francophone les raisons du succès de l'œuvre austenienne... et pourrait avoir comme objectif le plaisir du texte.

Cela signifie sortir du mythe victorien et reléguer une fois pour toutes au placard les teapots et bonnets à dentelles en reconnaissant notamment l'importance du paysage et de la nature dans les romans d'Austen conformément aux travaux récents qui explorent le dialogue qu'elle instaure avec le Romantisme (Tuite; Deresiewicz). Il est aussi impératif de renoncer aux mauvais procès adressés en bloc à la critique féministe et de réinscrire Austen dans l'histoire des romancières et dans celle des femmes au tournant du XIXe siècle (Johnson ; Kelly). Penser que ses héroïnes sont " résignées " parce qu'elles ne correspondent pas à ce qui définit l'émancipation et l'épanouissement d'une femme aujourd'hui constitue un raccourci indigne de la masse de connaissances accumulées depuis plus de trente ans sur le sujet. Il faut également reconnaître qu'Austen instaure un dialogue sérieux avec les auteurs et les idées de son temps. Un titre comme Sense and Sensibility ne doit pas se lire seulement comme un clin d'œil à la littérature édifiante et à ses raisonnements binaires, mais essentiellement comme un questionnement adressé aux Lumières et au Romantisme par une écrivaine ambitieuse (Knox-Shaw). Et puis, difficulté de poids, il faut tenir compte de ce que Janet Todd appelle, dans la « General Editor's Preface" de l'édition des presses universitaires de Cambridge, 
"Austen's exceptional cultural and literary position ». Dans la « langue culture " anglaise aujourd'hui globalisée, "Austen has a unique status among early English novelists - appreciated by the academy and the general public alike" (Todd : ix). Produire un texte susceptible de réunir un tel spectre de lecteurs en français peut bien apparaître comme la quadrature du cercle.

Enfin, moyen le plus sûr d'établir un lien entre les communautés interprétatives de "langue culture " anglaise et de "langue culture " française, il faut des traducteurs prêts à reconnaître la place de l'ironie au cœur du texte. Cette injonction est évidemment paradoxale puisque, au lieu de fournir une clé pour déchiffrer le sens du texte, elle en démultiplie les sens possibles. Si la tâche des retraducteurs s'annonce donc ardue, elle doit, pour réussir, s'appuyer d'avance sur un nombre de choix interprétatifs forts. En traduction, dit Berman, il faut toujours choisir. L'ironie d'Austen ne fait que rendre ce constat plus pressant. En s'appuyant sur la richesse de la critique austenienne d'expression anglaise mais aussi en tenant compte de l'enthousiasme que suscitent ses six romans en anglais tout autour du globe, le traducteur français devrait donc partir de l'a priori que ce que racontent ces textes ne sont pas des histoires de résignation et d'enfermement. Il faudrait trouver des retraducteurs qui, tout simplement, aiment le texte. En tant que critique des traductions, je laisserai donc parler ici, à titre d'exemple, ma propre subjectivité car il est temps que toute retraduction d'Austen parte de cette question élémentaire : qu'est-ce qui fait qu'on aime ses romans à ce point ? En tant que critique des traductions françaises, ma tâche consiste en partie à identifier ces éléments de l'œuvre qui m'ont attirée en anglais mais dont l'absence dans les traductions me déçoit. Pourquoi donc, avant même de devenir une lectrice universitaire de Sense and Sensibility, ai-je "croché" à ce roman comme rarement, malgré ma maîtrise alors chancelante de l'anglais?

La lectrice de vingt ans que j'étais a été frappée par l'énergie et l'intelligence des héroïnes austeniennes. Elinor Dashwood n'a jamais été pour moi un dragon de vertu mais une femme vivante, définie par 
sa profondeur et sa complexité, qui m’a émue à la fois par sa souffrance et par son humour, par sa force, et par le courage avec lequel elle embrasse bien malgré elle le rôle de chef de famille qui lui échoit à la mort de son père. À aucun moment je n'ai pensé que sa valeur exemplaire résidait dans ses bonnes manières. M'ont frappée son absence de naïveté et sa volonté, sa compassion également, et la conscience qu'elle a des souffrances que la société peut infliger à ceux qu'elle aime. Elle reste pour moi le personnage qui lance à sa sœur : "No, Marianne, never. My doctrine has never aimed at the subjection of the understanding " (SS : 108). Elle est celle qui connaît sa supériorité intellectuelle et renonce à la conversation idiote et suffisante de Robert Ferrars : "she did not think he deserved the compliment of rational opposition" (SS : 286). Elle est celle dont les émotions jaillissent littéralement à la fin du roman :

Elinor could sit no longer. She almost ran out of the room, and as soon as the door was closed, burst into tears of joy, which at first she thought would never cease. Edward, who had till then looked anywhere, rather than at her, saw her hurry away, and perhaps saw - or even heard, her emotion; for immediately afterwards he fell into a reverie, which no remarks, no inquiries, no affectionate address of Mrs Dashwood could penetrate, and at last, without saying a word, quitted the room, and walked out towards the village - leaving the others in the greatest astonishment and perplexity on a change in his situation, so wonderful and so sudden; - a perplexity which they had no means of lessening but by their own conjectures. (SS : 408).

Il me semble que le lecteur de Sense and Sensibility doit, comme Marianne et comme Edward, apprendre à lire Elinor et lui faire crédit des sentiments que les circonstances du roman l'empêchent d'exprimer. Il faut que la profondeur d'Elinor soit constamment présente à l'esprit du retraducteur. Comme Deidre Lynch l'a montré dans son étude des personnages chez Austen, Elinor est une héroïne définie par son intériorité : le respect des formes correspond à un "cover for privacy " et, chez elle, "self-effacement secures self-possession" (Lynch : 236). Or, d'Isabelle de Montolieu à Pierre Goubert, Elinor perd de sa substance pour devenir une de ces plates "pictures of perfection " qui, aux 
yeux d'Austen, constituaient le fléau des romans (Austen 1995 : 335). Dans la préface de 1815 à Raison et sensibilité, elle est décrite par Montolieu comme un «modèle » : "le plus parfait modèle pour les jeunes personnes » (cité dans Gilson : 152). Son sort n’a pas vraiment évolué puisque dans la présentation du roman dans la Pléiade en 2000, elle est encore dépeinte par Goubert comme « une héroïne sans relief » : «Elinor n'a pas assez de défauts ; elle est trop satisfaite d'ellemême ; elle est froide ; elle n'attire pas la sympathie" (Goubert : 1042). Le paratexte des traductions de Montolieu et Goubert est intéressant parce qu'il met en évidence l'importance du concept de " communauté interprétative " de Fish. À presque deux cents ans de distance, Montolieu et Goubert identifient une intention didactique à l'œuvre au cœur du roman. Du coup, chacun lit le texte sur le mode d'une opposition binaire simple entre Marianne et Elinor qui, chacune, refléterait un des termes du titre, qui est quant à lui beaucoup plus dichotomique en français qu'en anglais. C'est aussi à cette lecture binaire que nous convie la quatrième de couverture de l'édition 10/18: "Raison et sentiments sont joués par deux sœurs, Elinor et Marianne Dashwood. Elinor représente la raison, Marianne le sentiment. " Cette approche dichotomique de Sense and Sensibility est un trait saillant de la communauté interprétative de "langue culture » française qui contraste avec la communauté interprétative de "langue culture " anglaise. Pour cette dernière, une telle clarté n'est pas de mise. La critique se plaît à souligner, au contraire, à quel point Austen déroute ses lecteurs avec un titre qui repose sur une opposition illusoire entre les termes "sense" et "sensibility" (Tanner: 77 ; Armstrong : 3-4), termes qui, à l'époque où écrit Austen, constituent des nuances conceptuelles et non des contraires :

Contrasts are established through similitude rather than absolute difference. Ultimately, 'sense' and 'sensibility' are etymological relatives rather than linguistic strangers. (Ballaster : xviii)

Comment dès lors, première question pratique, résoudre l'épineuse question du titre ? Le français, il est vrai, n'offre pas la même palette 
de concepts que l'anglais. Ce qu'on observe, c'est que les titres retenus (Le Cour et la Raison, Raison et sentiments, Raison et sensibilité ou les deux manières d'aimer) prêtent implicitement au roman une structure binaire. Mais cette binarité implicite n'est pas seulement produite par des limitations de vocabulaire, elle est à chaque fois cautionnée par le paratexte puis reprise par la traduction. La communauté interprétative de «langue culture " française se distingue par son attachement à la structure binaire de Sense and Sensibility. Un retraducteur devrait donc ici chercher à se rapprocher de la communauté interprétative anglaise qui conçoit cette opposition comme un leurre, le titre comme un piège déjoué à la fois par l'étymologie des mots "sense " et "sensibility " et par la complexité du roman. Il me semble qu'une solution possible, au niveau du titre, consisterait à profiter de la popularité globale d'Austen pour, tout simplement, le maintenir en anglais, en misant sur le fait que Sense and Sensibility, comme une bonne chanson des Beatles, est aujourd'hui identifiable par le plus grand nombre. Le titre en anglais aurait aussi l'avantage de donner un peu de substance (et de mystère) à la catégorie " romancière anglaise " à laquelle la culture éditoriale française semble tenir.

Qu'en est-il maintenant d'Elinor et de la complexité du roman ? Sur cette question, je ne m'en tirerai pas à si bon compte, je le sais. Si j'étais retraductrice, mon impératif serait, à chaque page, de faire exister l'Elinor que j'ai décrite plus haut. L'exigence consiste également à me placer du point de vue de la communauté interprétative de "langue culture " anglaise et à me souvenir que l'ironie opère partout et à plusieurs échelles : au niveau d'un mot, d'une phrase, d'une plaisanterie insérée dans un dialogue comme au niveau de l'ensemble, qui est régi par le principe de l'ironie dramatique et du retournement des attentes des personnages et des lecteurs. Je dois donc me souvenir que " mon " Elinor est également le personnage dont le point de vue est celui qui se rapproche le plus de la narration et que son discours est, par conséquent, lui-même défini par l'ironie. Tels seraient mes choix interprétatifs de départ en fonction desquels je chercherais à produire mon texte en français. 
Pour conclure cet article, je commenterai certains passages clés à partir desquels reconstruire Elinor et sa relation à Marianne. Et je tenterai, en conséquence de mes commentaires, de proposer quelques exemples de retraduction de mon crû.

La première description comparée des sœurs Dashwood à la fin du premier chapitre constitue évidemment un endroit stratégique d'où entamer la révision de la lecture dichotomique du roman (SS : 7-8). La communauté interprétative à laquelle j'adhère me dit de l'aborder en évitant la note didactique ou alors en ironisant sur elle, m'enjoint aussi d'inscrire les personnages d'Elinor et de Marianne dans une intrigue qui va lier leurs aventures sentimentales respectives au débat culturel et philosophique de leur temps. Pour rompre avec la communauté existante de «langue culture " française, il faut, je pense, renoncer à des mots tels que " capables » et « incapables " et, généralement, tout faire pour éviter de poser Elinor en "modèle ". À ce stade le texte anglais présente les qualités des deux sœurs comme "naturelles " et désamorce les velléités de jugement en donnant une image également positive des qualités de Marianne ("She was generous, amiable, interesting: she was every thing but prudent ").

La première description d'Elinor postule chez elle une égale répartition des qualités du cœur et de l'esprit : l'un est aussi fort que l'autre, nous dit-on, sa raison à la mesure de ses émotions, et c'est parce qu'il en est ainsi qu'elle peut les "contrôler ». Pour rendre la nature émotionnelle de son personnage, souvent mise à mal en français, je dois notamment analyser les implications d'une expression aussi simple et directe que "her feelings were strong ". Il n'y a pas d'équivalent lexical français pour cette phrase, certes, mais il s'agit de réfléchir à la manière dont elle participe à l'univers austenien. L'expression des émotions vraies, chez Austen, dépend toujours d'un lexique simple, élémentaire, de termes tirés du registre courant qui évitent l'effet littéraire. À l'instar de Goubert, Castier et Privat, le retraducteur ne pourra peut-être pas éviter, en français, le mot "sentiment ", qui, à la différence de "feelings ", a une connotation littéraire forte. Mais il devra se souvenir que ce type de sobriété et de simplicité signale dans l'anglais d'Austen des 
personnages sincères, une propension à aimer qui émane du cœur et ne dépend d'aucune posture, d'aucun modèle culturel ou littéraire.

Ensuite, pour éviter la lecture didactique tout en permettant au roman de déployer son potentiel philosophique, je serais particulièrement attentive au mot "knowledge " dans la phrase : "it was a knowledge which her mother had yet to learn, and which one of her sisters had resolved never to be taught ". Cet énoncé résume, pour les tenants de la lecture didactique, l'horizon du roman. Comment en faire autre chose? Le mot "talent" retenu par Goubert (226) enferme le texte dans l'univers de la littérature féminine, au sens étroit et édifiant du terme. Le mot " connaissance" retenu par Castier (13) paraît trop anecdotique par rapport à l'enjeu philosophique réel, et le terme " science " choisi par Privat (10) instaure une distance ironique particulièrement mal placée dans la mesure où cet énoncé recouvre bel et bien une valeur fondamentale du roman. Il me semble que le mot "savoir " permettrait de quitter le registre de la littérature édifiante pour placer le roman sur le terrain de la morale au sens philosophique et épistémologique du terme. Ce qui distingue Elinor de Marianne, c'est sa capacité à lire non seulement des livres mais également " le grand livre ouvert " du monde tel qu'il se présente à elle. Le mot "savoir" reflète cette double qualité, livresque et empirique, de l'intelligence d'Elinor, à laquelle il confere une légitimité, voire une dignité, philosophique plutôt que didactique.

L'articulation entre son personnage et celui de sa sœur se joue sur le terme " eager " qualifiant Marianne : celle-ci a les mêmes qualités que sa sœur, nous dit-on, mais en plus "eager». Il y a donc une différence de degré entre les sœurs et non une différence de fond. Les mots " enthousiaste" (Goubert : 227) et "ardente" (Castier : 13) me paraissent judicieux, alors que le terme "passionnée " (Privat : 10) renforce la perception dichotomique des deux sœurs en sous-entendant qu'Elinor ne l'est pas. Enfin, dernier mot à retenir mon attention dans ce passage, le terme " romance " appliqué à Marianne dans la dernière phrase du chapitre. Contrairement aux trois traducteurs déjà cités, j'éviterais le terme "romanesque" pour la qualifier. Non pas 
parce qu'il s'agit d'une faute, mais parce qu'il inscrit Elinor et Marianne dans une dynamique didactique et féminine qui ne correspond pas à mon projet interprétatif. La lectrice romanesque est un topos qu'Austen explore dans ses Juvenilia et dans Northanger Abbey. Mais, comme Claudia Johnson et Janet Todd, je vois en Marianne une héroïne plus sérieuse, plus intense, plus cultivée et plus intelligente que Catherine Morland. Les valeurs de Marianne constituent un défi que ni le lecteur ni Elinor ne peuvent simplement balayer d'un revers de main. M'éloignant à cet endroit de la lettre du texte - pour peutêtre mieux la retrouver plus loin - j'utiliserais pour cette première description de Marianne le terme « idéalisme ", que je juge plus adapté à la nature sérieuse de son personnage de même qu'à ses lectures "romantiques" (au sens de l'histoire littéraire), essentiellement des poètes et des théoriciens de l'esthétique du paysage.

Le dialogue entre Marianne, Elinor et Edward au chapitre 17 est représentatif de la manière dont Austen mêle intrigue romanesque, psychologie des personnages et débat d'idées. La réponse vigoureuse d'Elinor citée plus haut ("No, Marianne, never. My doctrine has never aimed at the subjection of the understanding. ") relève à la fois de ce que l'on peut considérer comme une situation familiale - une dispute probablement récurrente entre les deux sœurs - et d'un postulat de l'auteur. Le mot "doctrine " rebondit sur le propos de Marianne, qui l'utilise de manière ironique et par provocation, mais, en même temps, il est parfaitement adapté au propos d'Elinor dont la vigueur ("never») constitue à la fois une riposte à la provocation de sa sœur et une défense de ses convictions, qui correspondent aux valeurs du roman. Par son caractère polymorphe, cet échange reflète aussi cette double qualité de l'œuvre d'Austen décrite par Todd: académique et grand public. L'énoncé philosophique (académique) s'insère dans ce qui est aussi une querelle entre "frangines ", avec laquelle le public peut s'identifier. Implicitement, Elinor fait comprendre à Marianne qu'elle aimerait bien, elle aussi, ne pas avoir à tenir toujours le mauvais rôle. La difficulté, ici, consiste à rendre cette conversation dans le cadre de cet implicite qu'est la relation entre les deux sœurs. Elle consiste à ne pas 
faire d'Elinor une pédante, ce qu'elle devient, malheureusement, dans toutes les retraductions (Castier : 113 ; Privat : 84 ; Goubert : 303). Toutes les retraductions sont "justes", mais aucune ne produit une Elinor proche de la communauté interprétative de langue anglaise car elles ne conservent que le contenu théorique de son propos en oblitérant la profondeur psychologique. Pour conserver celle-ci, il me semble qu'il faut éviter les tournures impersonnelles et/ou à la troisième personne. Il faudrait qu'Elinor s'adresse directement à sa sœur à la deuxième personne. Pour éviter l'impression de pédanterie, il faut peut-être renoncer au mot "doctrine" et faire en sorte qu'Elinor tutoie Marianne, alors que les textes hésitent entre vouvoiement (Goubert, Privat) et tutoiement (Castier). Il faut à cet endroit s'éloigner un peu de la lettre, en inversant peut-être l'ordre de certaines propositions :

Non, Marianne, jamais de la vie. Jamais je ne t'ai demandé de te soumettre à l'opinion des autres. Comment peux-tu me soupçonner de désirer l'assujettissement de l'intelligence ? La seule chose que j'ai essayé d'influencer chez toi, c'est ton comportement. Tu ne dois pas me faire dire ce que je n'ai jamais dit. Mon seul tort est d'avoir parfois soubaité, je l'avoue, que tu traites nos relations en général avec un peu plus d'égards, mais t'ai-je demandé une seule fois d'adopter leur point de vue sur des sujets sérieux?

Quant au commentaire sur le silence d'Elinor lors de son échange avec Robert Ferrars au chapitre 14 du deuxième volume (SS : 286 ; Castier : 284 ; Privat : 216 ; Goubert : 441), il est typique de la fusion que le texte opère régulièrement entre le point de vue de son personnage et celui de la narration. Cette fusion cautionne certes le personnage d'Elinor comme porteur des valeurs de l'auteur, ce qu'en retiennent les retraducteurs, mais il lui confere aussi, qualité capitale de son personnage aux yeux de la communauté interprétative de "langue culture " anglaise, la qualité d'observatrice ironique, qui correspond à la position du point de vue narratif en général. Un énoncé comme "Elinor agreed to it all, because she did not think he deserved the compliment of rational opposition " dévoile le côté "mordant" de son personnage, 
aux antipodes du "beau modèle " décrit par Montolieu et Goubert. Lépisode nous révèle une Elinor aussi capable que Marianne de manquer d'égard. Par le passage de la narration au discours indirect, la phrase glisse du point de vue du narrateur à celui d'Elinor, et finit par confondre les deux, créant un consensus parmi les lecteurs sur l'intelligence d'Elinor et la stupidité de Robert. L'énoncé est défini par l'humour qui lui-même dépend de la concision. Les propositions dans les retraductions sont, à cet égard, toutes trop longues. Non pas, je pense, parce que l'ironie d'Austen est en soi intraduisible, mais parce que même à cet endroit du texte, les retraducteurs préferent insister sur le côté « donneuse de leçon » d'Elinor. Tous choisissent de souligner l'activité de jugement en traduisant "she did not think" :

Elinor se déclara d'accord sur tout, car elle estimait qu'il ne méritait pas le compliment d'une opposition raisonnée. (Castier : 284)

Elinor convint de tout cela, car elle ne jugeait pas qu'il fût digne qu'on lui répondît sérieusement. (Privat: 216)

Elinor acquiesça à tout, étant donné qu'elle ne jugeait pas qu’il méritait le compliment d'une opposition rationnelle. (Goubert : 441).

À mes yeux, le "she did not think " ne sert qu'à introduire le discours indirect. Il ne nous invite pas à considérer Elinor comme pesant le pour et le contre. En tant que tel, et pour rendre le potentiel tranchant et ironique de l'énoncé, je l'abandonnerais purement et simplement et remplacerais le discours indirect par un discours indirect libre, qui est, après tout, une des innovations d'Austen :

Elinor se contenta de dire oui à tout car tenter de lui répondre aurait été lui faire trop de compliment.

La concision et l'humour sont aussi de mise chez Austen lorsqu'il s'agit de rendre les émotions. Au chapitre 12 du troisième volume, elle fait exploser en deux phrases seulement les sentiments qu'Elinor réprime tout au long du roman. L'intensité émotionnelle du passage - cité plus haut (SS : 408 ; Castier : 401 ; Privat : 307 ; Goubert : 538-39) - constitue le dénouement paradoxal d'un chapitre construit comme un grand 
moment comique : le personnage qui entre en scène, Edward, n'est pas celui que l'on attend (Brandon), et les premiers échanges entre lui et les femmes Dashwood sont fondés sur un qui pro quo. Face à l'embarras de la situation, Marianne et sa mère tentent de forcer leur naturel et de tenir pour une fois le rôle d'hôtesse polie habituellement dévolu à Elinor, jusqu'à ce que ce naturel revienne au galop, sitôt le malentendu dissipé, et qu'elles crient de concert: "'Mrs Robert Ferrars!'»(SS : 407). Les sentiments puissants mais cachés d'Elinor, que le roman annonce dès le premier chapitre, déboulent alors dans le texte dans un déchaînement quasi-hystérique. Mais, comparées à l'énergie du texte d'Austen, les retraductions me paraissent toutes convenues, et franchement pas drôles, empêtrées par la syntaxe dans des phrases trop longues. Il me parait plus important de choisir la concision comme principe réglant l'expression des émotions et de l'humour chez Austen, quitte à (shocking), laisser tomber un bout de phrase ("who had, till then, looked anywhere, rather than at her"). Les émotions sont également liées à un lexique simple, quasi élémentaire, qui, on l'a dit, évite la connotation littéraire. Elles dépendent d'énoncés factuels pour exprimer la singularité d'une situation : "Elinor could sit no longer ". Une expression comme "n'y tint plus" (Castier : 401 ; Privat : 307 ; Goubert : 538) est trop banale et trop abstraite. Enfin, une ironie (discrète) opère même à cet endroit le plus sentimental du roman: si Marianne et Mrs Dashwood s'avèrent fidèles à leur spontanéité légendaire, Elinor reste aussi ce qu'elle est et ne pleure qu'après avoir fermé la porte et, force de la raison oblige, parvient encore à plaisanter elle-même sur ces larmes contre lesquelles sa volonté est impuissante ("which at first she thought would never cease "):

Elinor ne pouvait plus rester en place. Elle se précipita presque en courant hors de la chambre et, sitôt la porte refermée, laissa éclater ses sanglots, des sanglots de joie qui, se dit-elle, ne s'arrêteraient jamais. Edward, qui jusque-là n'avait osé la regarder, la vit courir et peut-être vit - ou même entendit, son émotion; lui-même en tout cas fut immédiatement plongé dans une rêverie que ne purent interrompre ni 
remarque, ni question, ni aucune des expressions de tendresse que lui adressa Mrs Dashwood et, à la fin, il quitta la pièce sans dire un mot et sortit en direction du village - laissant tout loisir aux autres de sétonner et de s'exclamer sur son changement de situation si inattendu et si merveilleux, les abandonnant à une perplexité qui, pour diminuer, devait susciter un flot de conjectures.

Cette proposition ne constitue évidemment pas le mot de la fin, mais elle se veut une amorce qui, on l'espère, incitera de nouveaux traducteurs-lecteurs à faire exister en français une Austen qui ne soit pas le rabat-joie que la critique et les traductions nous proposent aujourd'hui. L'Austen que ses admirateurs aimeraient voir en français est celle qui récompense toujours, envers et contre le bon sens, l'énergie vitale, c'est le sens de ses happy endings. Même la raison d'Elinor, que traduction et retraductions présentent comme infaillible, ne suffit pas à contenir cette énergie du cœur : contre l'évidence et en dépit de la raison, elle s'imagine que ce sont ses cheveux qui se trouvent dans la bague d'Edward (SS : 114) et " in spite of herself, she had always admitted a hope" (SS : 404), un espoir que le roman finit par combler. Les larmes de joie d'Elinor, dont la justesse touche au cœur même les lecteurs les plus intellos, attendent encore d'être dites en français.

\section{valerie.cossy@unil.ch}

Valérie Cossy est professeure-assistante en littérature et en études genre à la Faculté des lettres de l'Université de Lausanne. Après des études à Lausanne et Oxford, elle a consacré sa thèse de doctorat (D. Phil.) aux premières traductions françaises des romans de Jane Austen. Elle a publié un livre (Jane Austen in Switzerland, A Study of the Early French Translations, Genève, Slatkine, 2006) ainsi que plusieurs articles sur le sujet. Ses recherches et ses publications portent notamment sur des figures de médiatrices culturelles telles qu'Isabelle de Charrière, Isabelle de Montolieu ou Frances Burney, et sur les enjeux symboliques et politiques de leur médiation. 


\section{Sources et traductions}

Austen, Jane, Jane Austen's Letters, ed. by Deirdre Le Faye, Oxford, Oxford University Press, 1995.

Austen, Jane, Sense and Sensibility, ed. by Edward Copeland, The Cambridge Edition of the Works of Jane Austen, Cambridge, Cambridge University Press, 2006.

Raison et sensibilité ou les deux manières d'aimer, traduit librement par Isabelle de Montolieu, Paris, Arthus Bertrand, 1815, 4 vol.

Le Cour et la Raison, traduit par Jules Castier, Lausanne, Marguerat, 1948.

Raison et sentiments, traduit par Jean Privat [1948], Paris, Christian Bourgois, collection 10/18, 1979.

Le Coeur et la Raison, traduit par Pierre Goubert, in Jane Austen, Euvres romanesques complètes, édition publiée sous la direction de Pierre Goubert, Paris, Gallimard, «Bibliothèque de la Pléiade », 2000, vol. I.

Raison et Sentiments, traduit de l'anglais par Isabelle de Montolieu et revu par Hélène Seyrès, Paris, l'Archipel, 1996.

Raison et sentiments, traduit de l'anglais par Isabelle de Montolieu et revu par Hélène Seyrès, Paris, l'Archipel, « Archipoche », 2006.

\section{Références critiques}

Armstrong, Isobel, Sense and Sensibility, London, Penguin, «Penguin Critical Studies ", 1994.

Ballaster, Ros, Introduction to Sense and Sensibility, London, Penguin, "Penguin Classics ", 1995, vii-xxv.

Berman, Antoine, Pour une critique des traductions : John Donne, Paris, Gallimard, "Bibliothèque des idées ", 1995 
Bickerton, David M., Marc-Auguste and Charles Pictet, the Bibliothèque britannique (1796-1815) and the Dissemination of British Literature and Science on the Continent, Genève, Slatkine, 1986.

Bour, Isabelle, "The Reception of Jane Austen's Novels in France ", dans Mandal, Anthony et Brian Southam ed., The Reception of Jane Austen in Europe, New York, London, Continuum, 2007.

Cossy, Valérie, Jane Austen in Switzerland, A Study of the Early French Translations, Genève, Slatkine, 2006.

Deresiewicz, William, Jane Austen and the Romantic Poets, New York, Columbia University Press, 2004.

Fish, Stanley, "What Makes an Interpretation Acceptable ? », dans son livre Is There a Text in this Class? The Authority of Interpretative Communities, Cambridge (Mass), Harvard University Press, 1980, p. 338-55.

Gilson, David, A Bibliography of Jane Austen, Oxford, Clarendon Press, 1982, p. x-xy consacrées aux traductions.

Jordis, Christine, "Une littérature féminine anglaise ? ", Magazine littéraire, $\mathrm{n}^{\circ}$ 476, juin 2008, p. 56-59.

Johnson, Claudia L., Jane Austen, Women, Politics and the Novel, Chicago, University of Chicago Press, 1988.

Johnson, Claudia L., "'A Sweet Face as White as Death' : Jane Austen and the Politics of Female Sensibility", Novels XXII (1989) : 159-174.

Kelly, Gary, "Jane Austen, Romantic Feminism, and Civil Society ", in Jane Austen and Discourses of Feminism, ed. by Devoney Looser, London, Macmillan, 1995, 19-34.

Kirkham, Margaret, Jane Austen, Feminism and Fiction, Brighton, Harvester, 1983.

Knox-Shaw, Peter, Jane Austen and the Enlightenment, Cambridge, Cambridge University Press, 2004. 
Lynch, Deidre Shauna, The Economy of Character, Novels, Market Culture, and the Business of Inner Meaning, Chicago, University of Chicago Press, 1998.

Parent-Lardeur, Françoise, Lire à Paris au temps de Balzac, Les cabinets de lecture à Paris, 1815-1830, Paris, Éditions de l'École des Hautes Études en Sciences Sociales, 1981.

Sutherland, Kathryn, introduction à James Edward Austen-Leigh, A Memoir of Jane Austen and Other Family Recollections, Oxford, Oxford University Press, 2002.

Tanner, Tony, Jane Austen, London, Macmillan, 1986.

Todd, Janet, The Cambridge Introduction to Jane Austen, Cambridge, Cambridge University Press, 2006.

Todd, Janet, "General Editor's Preface ", The Cambridge Edition of the Works of Jane Austen, Sense and Sensibility, ed. by Edward Copeland, Cambridge, Cambridge University Press, 2006, ix-xii.

Trunel, Lucile, "Les éditions françaises de l'œuvre de Jane Austen (1815-2007) : l'apport de l'histoire éditoriale à la compréhension de la réception de l'auteur en France ", thèse de doctorat, Université Paris Diderot (Paris 7), 2008.

Tuite, Clara, Romantic Austen, Sexual Politics and the Literary Canon, Cambridge, Cambridge University Press, 2002. 